\title{
EFFECT OF HIGHER APPROXIMATION OF KRYLOV- -BOGOLIUBOV-MITROPOLSKII'S SOLUTION AND MATCHED ASYMPTOTIC SOLUTION OF A DIFFERENTIAL SYSTEM WITH SLOWLY VARYING COEFFICIENTS AND DAMPING NEAR TO A TURNING POINT
}

\author{
Roy K. C. and Shamsul Alam M. \\ Dept. of Math, Rajshahi University of Engineering \\ and Technology. Rajshahi 6204, Bangladesh
}

\begin{abstract}
Second approximate solution of a second order differential equation with slowly varying coefficients and damping is obtained by Krylov-Bogoliubov-Mitropolskii method The method is illustrated by an example. The second or higher order approximate solution is able to give better results than first approximate solution when the reduced frequency is many times larger than the small parameter. On the contrary, higher order solution diverges faster than the lower order solution when the reduced frequency becomes small (i.e., near to a turning point). In these situations matched asymptotic solution is important. An example is made to illustrate the matter.
\end{abstract}

\section{Introduction}

There are some well known perturbation methods (e.g., Poincare method [1], WKB method [2-4], two-scale method [5-6] or Krylov-Bogoliubov-Mitropolskii (KBM) method [7-9] for handling linear and nonlinear differential systems involving slowly varying coefficients. Among the above procedures, KBM method is convenient and is widely used. The method has been extended to damped oscillatory and purely non-oscillatory systems with slowly varying coefficients by Bojadziev and Edwards [10]. Recently, Shamsul [11] has presented a brief way to determine KBM solution (first order) of an $n$-th, $n=2,3, \cdots$ order differential system. In an another recent paper, Shamsul et al [12] have presented an asymptotic solution of a second order differential system in presence of strong linear damping force based on [13]. Sometimes first approximate solution obtained in [10-11] gives desired result when the linear damping effect is very small. Otherwise the solution (even if the damping is small rather than very small) gives incorrect result after a time interval, $T>>1$ where the reduced frequency becomes small. It is interesting to note that higher approximate solutions are unable to give correct result in these cases, since they diverge faster than first order or a lower order solution. In these situations matched asymptotic solution is only able to give desired result. Shamsul et al [14] found an asymptotic of a second order equation characterized by purely non-oscillatory process. When reduced frequency becomes 
small, the oscillation is almost non-oscillatory or purely non-oscillatory, so that Shamsul et $a l$ 's [14] solution is possible to match with KBM [9-11] solution in these situations.

\section{The Method}

Bojadziev and Edwards [10] have considered the following second order ordinary nonlinear differential equation with slowly varying coefficients

$$
\ddot{x}+2 k(\tau) \dot{x}+\nu^{2}(\tau) x=\varepsilon f(x, \dot{x}, \tau),
$$

and obtained a first approximate solution of Eq. (2.1). But we can easily obtain the second approximation of Eq. (2.1) followed by Popov [15], who actually determined a second approximate solution of a second order equation [similar to Eq. (2.1)] with constant coefficients and significant damping, i.e., $k>\varepsilon$. But in the case of variable coefficients, an extended form KBM solution $[7-10,15]$ is needed even if the damping is small, i.e., $k=\mathrm{O}(\varepsilon)$ especially when $\nu(\tau)$ is in a decreasing order.

For $\varepsilon=0$, Eq. (2.1) has two eigen-values namely $-k\left(\tau_{0}\right) \pm i \omega\left(\tau_{0}\right), \omega^{2}\left(\tau_{0}\right)=\nu^{2}\left(\tau_{0}\right)-$ $k^{2}\left(\tau_{0}\right)(\omega(\tau)$ is known as a reduced frequency) and the unperturbed solution of Eq. (2.1) becomes

$$
x(t, 0)=a_{0} e^{-k\left(\tau_{0}\right) t} \cos \left[\omega\left(\tau_{0}\right) t+\varphi_{0}\right],
$$

where $a_{0}$ and $\varphi_{0}$ are arbitrary constants and $\tau_{0}$ represents the value of $\tau$ when $\varepsilon=0$.

When $\varepsilon \neq 0$, there exist an asymptotic solution of Eq. (2.1) in the form [9-12]

$$
x(t, \varepsilon)=a \cos \psi+\varepsilon u_{1}(a, \psi, \tau)+\varepsilon^{2} u_{2}(a, \psi, \tau)+\varepsilon^{3} \cdots,
$$

where $a$ and $\psi$ satisfy the differential equations [10-12]

$$
\begin{aligned}
& \dot{a}=-k(\tau) a+\varepsilon A_{1}(a, \tau)+\varepsilon^{2} A_{2}(a, \tau)+\varepsilon^{3} \cdots, \\
& \dot{\psi}=\omega(\tau)+\varepsilon B_{1}(a, \tau)+\varepsilon^{2} B_{2}(a, \tau)+\varepsilon^{3} \cdots .
\end{aligned}
$$

To determine the unknown functions $u_{1}, u_{2}, \cdots, A_{1}, A_{2}, \cdots ; B_{1} B_{2}, \cdots$, it was early restricted in KBM method [7-9] that the functions $u_{1}, u_{2}, \cdots$ exclude first harmonic terms and $A_{1}, A_{2}, \cdots ; B_{1} B_{2}, \cdots$ are independent of phase variable $\psi$. It is noted that expansion Eq. (2.3) is valid when $\frac{\dot{a}}{a}<<\nu(\tau)$ and $\dot{\varphi}<<\nu(\tau)$ or $\dot{\psi}-\omega<<\nu(\tau)$ [16]. Certainly these restrictions are strictly maintained when the coefficients, $k, \nu$ or $\omega$ are constants. On the contrary, it is too much difficult to maintain the later restriction when $\nu(\tau)$ or $\omega(\tau)$ is in a decreasing order.

Differentiating Eq. (2.3) twice with respect to $t$, substituting for the derivatives $\dot{x}, \ddot{x}$ and $x$ into Eq. (2.1), utilizing Eq. (2.4) and comparing the coefficients of $\varepsilon, \varepsilon^{2}$, we obtain

$$
\begin{aligned}
& \omega_{0}^{\prime} a \sin \psi-k^{\prime} a \cos \psi-\left(k a \frac{\partial A_{1}}{\partial a}-k A_{1}-2 \omega a B_{1}\right) \cos \psi-\left(2 \omega A_{1}-k a^{2} \frac{\partial B_{1}}{\partial a}\right) \sin \psi \\
& +\left(\left(-k a \frac{\partial}{\partial a}+\frac{\partial}{\partial \psi}+k\right)^{2}+\nu^{2}-k^{2}\right) u_{1}=f(a \cos \psi,-k a \cos \psi-\omega a \sin \psi)
\end{aligned}
$$




$$
\begin{aligned}
& \left(k a \frac{\partial A_{2}}{\partial a}-k A_{2}-2 \omega a B_{2}\right) \cos \psi-\left(2 \omega A_{2}-k a^{2} \frac{\partial B_{2}}{\partial a}\right) \sin \psi+ \\
& \left(\left(-k a \frac{\partial}{\partial a}+\frac{\partial}{\partial \psi}+k\right)^{2}+\nu^{2}-k^{2}\right) u_{2}=u_{1} f_{x}(\cdot)+ \\
& +\left(-k a \frac{\partial u_{1}}{\partial a}+\omega \frac{\partial}{\partial \psi}+A_{1} \cos \psi-a B_{1} \sin \psi\right) f_{\dot{x}}(\cdot)+2 k a A_{1} \frac{\partial^{2} u_{1}}{\partial a^{2}}+ \\
& +2\left(k a B_{1}-\omega A_{1}\right) \frac{\partial^{2} u_{1}}{\partial a \partial \psi}-2 \omega B_{1} \frac{\partial u_{1}}{\partial \psi}+\left(k a \frac{\partial A_{1}}{\partial a}-k A_{1}\right) \frac{\partial u_{1}}{\partial a}+k a \frac{\partial B_{1}}{\partial a} \frac{\partial u_{1}}{\partial \psi}- \\
& -2 k B_{1} \frac{\partial u_{1}}{\partial \psi}+\left(-A_{1}^{\prime}-A_{1} \frac{\partial A_{1}}{\partial a}+a B_{1}^{2}\right) \cos \psi+\left(B_{1}^{\prime}+A_{1} \frac{\partial B_{1}}{\partial a}+2 A_{1} B_{1}\right) \sin \psi
\end{aligned}
$$

When the coefficients of Eq. (2.1) become constants, $k^{\prime}=\omega^{\prime}=A_{1}^{\prime}=B_{1}^{\prime}=0$, and Eqs. (2.5)-(2.6) take the forms of those obtained by Popov [15] (see also [17]). Clearly the terms with appear in Eqs. (2.5)-(2.6) due to change of the coefficients in Eq. (2.1). Sometimes a first order solution with constant coefficients agrees with numerical solution nicely while corresponding first order solution with variable coefficients gives desired results for a particular time interval, so that the problem is in its linear part only. Therefore our investigation may be limited to the linear part of Eq. (2.1), i.e., we would find the terms of a second approximate solution which are appeared for changing the coefficients of Eq. (2.1). However, in the case of linear system, $f(a \cos \psi,-k a \cos \psi-\omega a \sin \psi)$, i.e., $f(. \cdot)$ contains terms involving $a \cos \psi, a \sin \psi$, i.e., $f$ is free from $\cos 2 \psi, \sin 2 \psi, \sin 3 \psi, \ldots$ Thus $u_{1}=0$ and Eqs. (2.5)-(2.6) reduce to the simplest form as

$$
\begin{gathered}
\omega_{0}^{\prime} a \sin \psi-k^{\prime} a \cos \psi+2 \omega a B_{1} \cos \psi-2 \omega A_{1} \sin \psi=f, \\
-2 \omega a B_{2} \cos \psi-2 \omega A_{2} \sin \psi-\left(A_{1}^{\prime}+A_{1} \frac{\partial A_{1}}{\partial a}-a B_{1}^{2}\right) \cos \psi+ \\
\left(B_{1}^{\prime}+A_{1} \frac{\partial B_{1}}{\partial a}+2 A_{1} B_{1}\right) \sin \psi=0 .
\end{gathered}
$$

In general, $f(\cdot), u_{1}, u_{2}$ be expanded in a Fourier series as [7-9]

$$
f(\cdot \cdot)=\sum_{n=0}^{\infty} F_{n}(a) \cos n \psi+G_{n}(a) \sin n \psi,
$$

and that $u_{1}, u_{2}$ have the terms in $\cos \psi$ and $\sin \psi$ missing, so that

$$
\begin{aligned}
& u_{1}=U_{0}(a, \tau)+\sum_{n=2}^{\infty} U_{n}(a, \tau) \cos n \psi+V_{n}(a, \tau) \sin n \psi \\
& u_{2}=P_{0}(a, \tau)+\sum_{n=2}^{\infty} P_{n}(a, \tau) \cos n \psi+Q_{n}(a, \tau) \sin n \psi
\end{aligned}
$$


since $k^{\prime}=0$. Now equating the coefficients of $\cos \psi, \sin \psi$ we obtain

$$
A_{1}=-\frac{\omega^{\prime} a}{2 \omega}, \quad B_{1}=0
$$

Then substituting the values of $A_{1}$ and $B_{1}$ from Eq. (3.5) into Eq. (2.8), we obtain

$$
-2 \omega a B_{2} \cos \psi-2 \omega A_{2} \sin \psi-\left(-\frac{d}{d \tau}\left(\frac{\omega^{\prime}}{2 \omega}\right)+\left(\frac{\omega^{\prime}}{2 \omega}\right)^{2}\right) a \cos \psi=0 .
$$

Equating again the coefficients of $\cos \psi, \sin \psi$ we obtain

$$
A_{2}=0, \quad B_{2}=\frac{1}{2 \omega}\left(-\frac{d}{d \tau}\left(\frac{\omega^{\prime}}{2 \omega}\right)+\left(\frac{\omega^{\prime}}{2 \omega}\right)^{2}\right) .
$$

Substituting the values of $A_{1}, B_{1}, A_{2}, B_{2}$ from Eqs. (3.5) and (3.7) into Eq. (2.4), we obtain

$$
\dot{a}=-\varepsilon a-\frac{\varepsilon \omega^{\prime} a}{2 \omega}, \quad \dot{\psi}=\omega+\frac{\varepsilon^{2}}{2 \omega}\left(-\frac{d}{d \tau}\left(\frac{\omega^{\prime}}{2 \omega}\right)+\left(\frac{\omega^{\prime}}{2 \omega}\right)^{2}\right) .
$$

Eq. (3.8) has an exact solution. Let us consider a particular case when $l_{0}=g$, so that $\omega^{2}=e^{-\tau}-\varepsilon^{2}$. Integrating Eq. (3.8) with respect to $t$, yields

$$
\begin{aligned}
& a=a_{0} e^{-\varepsilon t} \sqrt{\frac{\omega_{0}}{\omega}} \\
& \psi=\psi_{0}+\frac{2}{\varepsilon}\left(\omega_{0}-\omega\right)+2 \tan ^{-1} \frac{\varepsilon\left(\omega-\omega_{0}\right)}{\left(\varepsilon^{2}+\omega_{0} \omega\right)}+\frac{\varepsilon}{16}\left(\frac{1}{\omega_{0}}-\frac{1}{\omega}\right) \\
& +5 \frac{\varepsilon^{3}}{48}\left(\omega^{-3}-\omega_{0}^{-3}\right),
\end{aligned}
$$

where $\omega_{0}^{2}=1-\varepsilon^{2}$. Thus an approximate solution of Eq. (3.1) is

$$
x=a \cos \psi,
$$

where $a$ and $\psi$ are given by Eq. (3.9).

Now we have to solve Eq. (2.5) for obtaining a first order approximation of Eq. (3.2), when $\varepsilon f=\nu^{2} \frac{x^{3}}{6}$. But in the case of small or significant or large damping effect, first order solution of a pendulum with constant coefficients and with slowly varying coefficients have already been obtained in $[11,18]$. So, we are not interested to repeat it. From a unified solution obtained in [11], we easily get the desired solution (in order of $\varepsilon$ ) as

$$
x \approx a \cos \psi-\frac{a^{3}}{192} \cos 3 \psi
$$

where $a$ and $\psi$ satisfy the following equations

$$
\dot{a} \approx-\varepsilon a-\frac{\varepsilon \omega^{\prime} a}{2 \omega}-\frac{\varepsilon a^{3}}{16}, \quad \dot{\psi} \approx \omega\left(1-\frac{a^{2}}{16}\right) .
$$


and

$$
\frac{\partial \tilde{u}}{\partial t}+c \tilde{u}=\sum_{r=2}^{\infty} F_{r} \beta^{r} .
$$

The particular solution of Eqs. (2.16)-(2.18) gives the unknown functions $\tilde{A}_{1}, \tilde{B}_{1}$ and $\tilde{u}$, which complete the determination of first order solution of Eq. (2.11). The method can be carried out to higher orders in a similar way, but the first order solution is able to give desired results for $t \geq T$.

\section{Example}

To illustrate the method, we consider the oscillations of a pendulum with variable length. The differential equation of the motion is $[11,18]$

$$
\frac{d}{d t}\left(m l^{2}(\tau) \dot{x}\right)+m g l(\tau) \sin x=0
$$

where $m$ is the mass, $x$ the angle of deviation of the pendulum from the vertical, $g$ the acceleration of gravity, $l(\tau)$ the length of the pendulum varying slowly with time. For small oscillations we use the first term of the development of $\sin x$, while we may use first two terms of $\sin x$ when $x=O(1)$. In these situations, Eq. (3.1) can be written as

$$
\ddot{x}+2 \varepsilon l^{\prime}(\tau) \frac{\dot{x}}{l(\tau)}+\nu^{2}(\tau) x=0,
$$

or,

$$
\ddot{x}+2 \varepsilon l^{\prime}(\tau) \frac{\dot{x}}{l(\tau)}+\nu^{2}(\tau) x=\nu^{2}(\tau) \frac{x^{3}}{6},
$$

where $\nu^{2}(\tau)=\frac{g}{l(\tau)}, \tau=\varepsilon t, l^{\prime}=\frac{d l}{d \tau}$. Generally the solution of Eq. (3.2) or Eq. (3.3) are determined by assuming that $l$ changes linearly with $\tau[10-11,18]$, but in this paper we consider the simplest case when $l(\tau)=l_{0} e^{\tau}, l_{0}$ constant. Therefore, $\frac{l^{\prime}}{l}=1$ and the damping is purely constant. Eq. (3.2) is similar to that of an aging spring [19-20] in presence of a viscous force. It is noted that Hung and Wu [19] obtained an exact solution of Eq. (3.2) in terms of Bessel's functions when $-2 \varepsilon \dot{x}$ is missing. Similar exact solution exists even if the linear damping, $-2 \varepsilon \dot{x}$ is present (see Sec. 5). However perturbation techniques are not constructed in terms of Bessel's functions to date. Therefore, it is not possible to solve nonlinear equation Eq. (3.3) in terms of Bessel's functions on the basis of a perturbation technique. It is interesting to note that Eq. (3.1) has an exact solution [in terms of Elliptic functions (Jacobian)] when $l$ is constant and a perturbation solution can be found when the damping is occurred due to a small viscous force (see [16] for details). But in our problem (concern of this paper), the small linear damping force, $-2 \varepsilon \dot{x}$ is added to the motion due to increasing length (in order of $e^{\varepsilon t}$ ) of the pendulum. In this case the method developed in [16] is useless.

In Eq. (3.2), $k=\varepsilon, \omega^{2}=\frac{e^{-\tau} g}{l_{0}}-\varepsilon^{2}$ and $f=0$. Therefore Eq. (2.7) readily becomes

$$
\omega_{0}^{\prime} a \sin \psi+2 \omega a B_{1} \cos \psi-2 \omega A_{1} \sin \psi=0
$$




$$
e^{-2 \varepsilon t}\left(\frac{\partial \tilde{B}_{1}}{\partial t}-2 \varepsilon \tilde{B}_{1}\right)=-\frac{\beta}{\varepsilon} e^{-\varepsilon T-\varepsilon t} e^{-2 \varepsilon t}
$$

and

$$
\frac{\partial \tilde{u}}{\partial t}+c \tilde{u}=0
$$

The particular solutions of Eqs. (3.17)-(3.19) are

$$
\tilde{A}_{1}=-\frac{\alpha}{\varepsilon^{2}} e^{-\varepsilon T-\varepsilon t}, \quad \tilde{B}_{1}=\frac{\beta}{3 \varepsilon^{2}} e^{-\varepsilon T-\varepsilon t},
$$

and $\tilde{u}=0$. From Eq. (3.20), we see that perturbation solution Eq. (2.12) is certainly valid when $e^{-\varepsilon T}<\varepsilon^{2}$. Now substituting the values of $\tilde{A}_{1}, \tilde{B}_{1}$ from Eq. (3.20) into Eq. (2.13) and integrating with respect to $t$, we obtain

$$
\alpha=\alpha_{0} \exp \left[\frac{e^{-\varepsilon T}\left(e^{-\varepsilon t}-1\right)}{\varepsilon^{2}}\right], \quad \beta=\beta_{0} \exp \left[\frac{e^{-\varepsilon T}\left(1-e^{-\varepsilon t}\right)}{3 \varepsilon^{2}}\right] .
$$

Thus the first order solution of the linear Eq. (3.2) is

$$
x(t, \varepsilon)=\alpha+\beta e^{-2 \varepsilon t},
$$

where $\alpha$ and $\beta$ are given by Eq. (3.19). Solution Eq. (3.22) is a first approximate solution of Eq. (3.2) and it may be used as an approximate solution of Eq. (3.3) when $t \geq T$, while solution Eq. (3.10) or Eq. (3.11) is used when $t \leq T$.

\section{Results and Discussions}

Usually higher approximate solution is used for obtaining better results. But in the case of varying coefficients, sometimes higher approximations give more incorrect results. Solution Eq. (3.11) together with Eqs. (3.14)-(3.15) represents the first approximation while Eq. (3.11) represents with Eqs. (3.14), (3.16) represents second approximation. Comparing Eqs. (3.15) and (3.16) we may conclude that second approximation gives more incorrect results than the first approximation, since $\psi$ given in Eq. (3.16) becomes large as that of in Eq. (3.15) when $\omega=\mathrm{O}(\varepsilon)$. Clearly, in Eqs. (3.15)-(3.16) $\psi$ relates with $\omega$ as respectively $\omega^{-1}$ and $\omega^{-3}$. Therefore, second approximation gives more incorrect results than the first approximation of the phase $\psi$, which makes $x$ incorrect (see Fig. 1). Thus the matched asymptotic solution is needed in this situation. These solution gives desired results (see Fig. 2).

\section{Exact solution of Eq. (3.2)}

For the particular case when $g=l_{0}$, Eq. (3.2) becomes

$$
\ddot{x}+2 \varepsilon \dot{x}+e^{-\tau} x=0 .
$$


It is obvious that solution Eq. (3.10) together Eq. (3.8) or Eq. (3.9) represents a second approximate solution of Eq. (3.2). On the contrary, solution Eq. (3.11) together with Eq. (3.12) represents first approximate solution of Eq. (3.3) while Eq. (3.22) represents a mixed approximate solution of Eq. (3.3) [in which second approximation is for the linear part while first approximation is for the nonlinear part] where $a$ and $\psi$ satisfy the following equátions

$$
\dot{a}=-\varepsilon a-\frac{\varepsilon \omega^{\prime} a}{2 \omega}-\frac{\varepsilon a^{3}}{16}, \quad \dot{\psi}=\omega\left(1-\frac{a^{2}}{16}\right)+\frac{\varepsilon^{2}}{2 \omega}\left(-\frac{d}{d \tau}\left(\frac{\omega^{\prime}}{2 \omega}\right)+\left(\frac{\omega^{\prime}}{2 \omega}\right)^{2}\right) .
$$

Eqs. (3.12) or Eq.s (3.13) has not an exact solution. Usually the integration of Eqs. (2.4) is done by well-known techniques of calculus; but sometimes they are solved by a numerical procedure [21-22]. In this case, the perturbation method facilitates the numerical method. The variables $a$ and $\psi$ change slowly with time. So, it requires the numerical calculation of a few number of points. Contrary, a direct attempt to solve Eq. (2.11) requires the numerical calculation of a great number of points. Often one is not interested in only the oscillating processes itself, i.e., finding the $x$ in terms of $t$, but mainly in the behavior of the amplitude, $a$ and the phase $\varphi$ or $\psi$, which as $t$ increases characterize the oscillating processes [11,22]. However, an approximate solution of Eq. (3.12) or Eq. (3.13) may be found easily by considering the third term of the first equation, namely $\frac{\varepsilon a^{3}}{16} \approx \frac{\varepsilon a a_{0}^{2} e^{-2 \varepsilon t}}{16}$, since the change of $a$ is small. Thus the approximate solution of the first equation of Eq. (3.12) or Eq. (3.13) is

$$
a \approx a_{0} e^{-\varepsilon t}\left(\sqrt{\frac{\omega_{0}}{\omega}}+\frac{a_{0}^{3}}{32}\left(e^{-2 \varepsilon t}-1\right)\right) .
$$

Now substituting $a=a_{0} e^{-\varepsilon t} \sqrt{\frac{\omega_{0}}{\omega}}$ (neglecting the second term) into second member of Eq. (3.12) or Eq. (3.13) and integrating with respect to $t$, we obtain

$$
\psi \approx \psi_{0}+\frac{2}{\varepsilon}\left(\omega_{0}-\omega\right)+2 \cdot \tan ^{-1} \frac{\varepsilon\left(\omega-\omega_{0}\right)}{\left(\varepsilon^{2}+\omega_{0} \omega\right)}+\frac{a_{0}^{2}}{32 \varepsilon}\left(e^{-2 \varepsilon t}-1\right),
$$

or

$$
\begin{aligned}
& \psi \approx \psi_{0}+\frac{2}{\varepsilon}\left(\omega_{0}-\omega\right)+2 \tan ^{-1} \frac{\varepsilon\left(\omega-\omega_{0}\right)}{\left(\varepsilon^{2}+\omega_{0} \omega\right)}+\frac{a_{0}^{2}}{32 \varepsilon}\left(e^{-2 \varepsilon t}-1\right) \\
& +\frac{\varepsilon}{16}\left(\omega_{0}^{-1}-\omega^{-1}\right)+\frac{5 \varepsilon^{3}}{48}\left(\omega^{-3}-\omega_{0}^{-3}\right) .
\end{aligned}
$$

Clearly solution Eq. (3.10) or Eq. (3.11) is valid when $\omega>\varepsilon$; but the perturbation solution Eq. (3.10) or Eq. (3.11) is unable to give desired results when $\omega=O(\varepsilon)$. In this case we have to use a solution of the type Eq. (2.12) instead of Eq. (3.10) or Eq. (3.11). Since we have already considered the case where $\nu^{2}(t+\varepsilon T) x<<1$, we may neglect the term $\nu^{2}(t+\varepsilon T) \frac{x^{3}}{6}$. Therefore, we solve linear Eq. (3.2) instead of Eq. (3.3). In this situation Eqs. (2.16)-(2.18) become

$$
\frac{\partial \tilde{A}_{1}}{\partial t}+2 \varepsilon \tilde{A}_{1}=-\frac{\alpha}{\varepsilon} e^{\varepsilon T+\varepsilon t}
$$




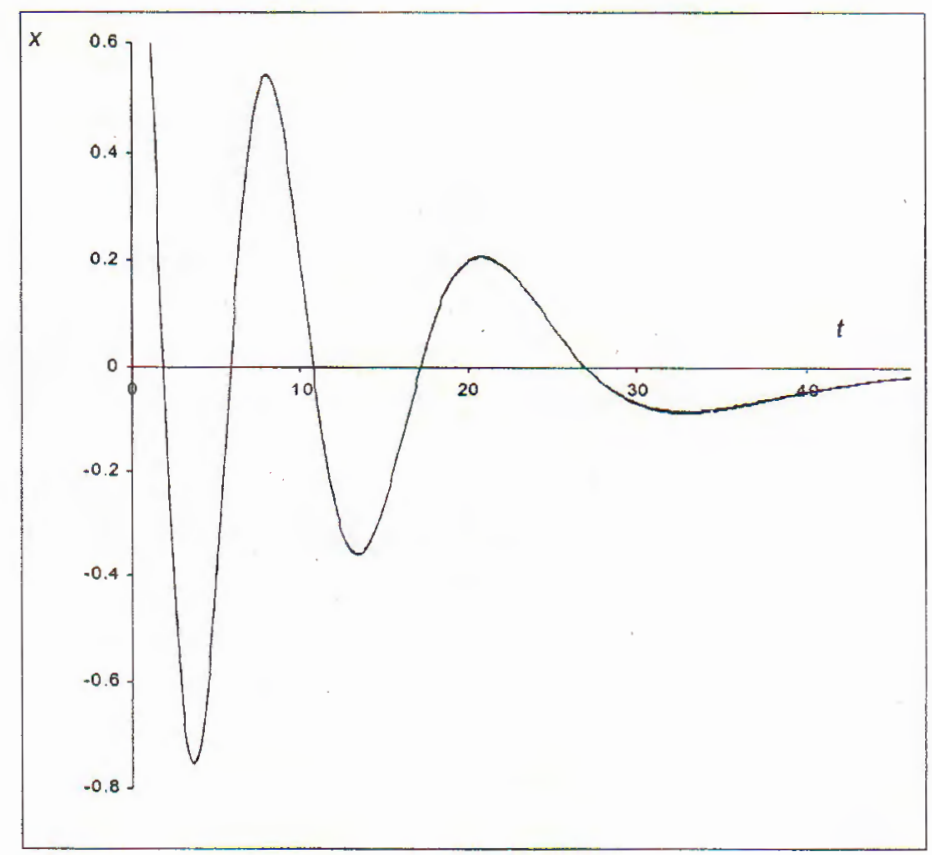

Fig. 2

Second approximate (mixed type: second approximation is for the linear part and first approximation for the nonlinear part) solution Eq. (3.11) of Eq. (3.3) in which $\psi$ is computed by Eq. (3.16) for $\varepsilon=0.1$ [with initial conditions, $x(0)=1, \dot{x}(0)=0$ or $a(0)=$ $1.0079, \dot{\psi}(0)=-0.0738]$ is plotted when $t \leq 38$, where $x(38)=-0.0605, \dot{x}(38)=0.0071$ and then initialized these values of $x, \dot{x}$, approximate solution Eq. (3.22) is computed and potted from $t=38$ to $t=46$. The corresponding numerical solution is also plotted to compare the results. The figure indicates that the matched solution nicely agrees with the numerical solution.

\section{References}

1. Poincare H. Les Methods Nouvelles de la Mecanique Celeste, Paris, 1892.

2. Wetzel, G. Z. Physik 38 (1926) 518.

3. Kramers H. A., G. Z. Physik 39 (1926) 828.

4. Brillouin L., Compt. Rend. 183 (1926) 24.

5. Feshcnko S. F., Shkil N. I. and Nikolenko L. D., Asymptotic Method in the Theory of linear differential Equation,.(in Russian); Naukova Dumka, Kiev 1966 [English translation, Amer, Elsevier Publishing Co., INC. New York 1967).

6. Nayfeh A. H., Perturbation Methods, J. Wiley, New York, 1973.

7. Krylov N. N. and N., N., Bogoliubov, Introduction to nonlinear Mechanics. Princeton University presses, New Jersey, 1947.

8. Bogoliubov, N. N. and Yu. Mitropolskii, Asymptotic Methods in the Theory of nonlinear Oscillations, Gordan and Breach, New York, 1961.

9. Mitropolskii Yu., Problems on Asymptotic Methods of non-stationary Oscillations (in Russian), Izdat, Nauka, Moscow, 1964. 
In absence of damping, Hung and Wu [19] presented an exact solution of $\ddot{x}+e^{-\tau} x=0$ in terms of Bessel's functions, introducing the transformation

$$
s=2 \varepsilon^{-1} e^{-\frac{\varepsilon t}{2}} .
$$

However, we can able to present an exact solution of Eq. (5.1) replacing $t$ by $s$ as

$$
\frac{d^{2} x}{d s^{2}}-\frac{3}{s} \frac{d x}{d s}+x=0 .
$$

Eq. (5.3) represents a modified Bessel's equation (of the form $x y^{\prime \prime}+(1-2 n) y^{\prime}+x y=0$ ), whose solution is

$$
x=s^{2}\left[A J_{2}(s)+B Y_{2}(s)\right],
$$

where $A$ and $B$ are two arbitrary constants. It is noted Hung and $\mathrm{Wu}$ 's [19] solution for the equation, $\ddot{x}+e^{-\tau} x=0$ is $x=A J_{0}(s)+B Y_{0}(s)$.

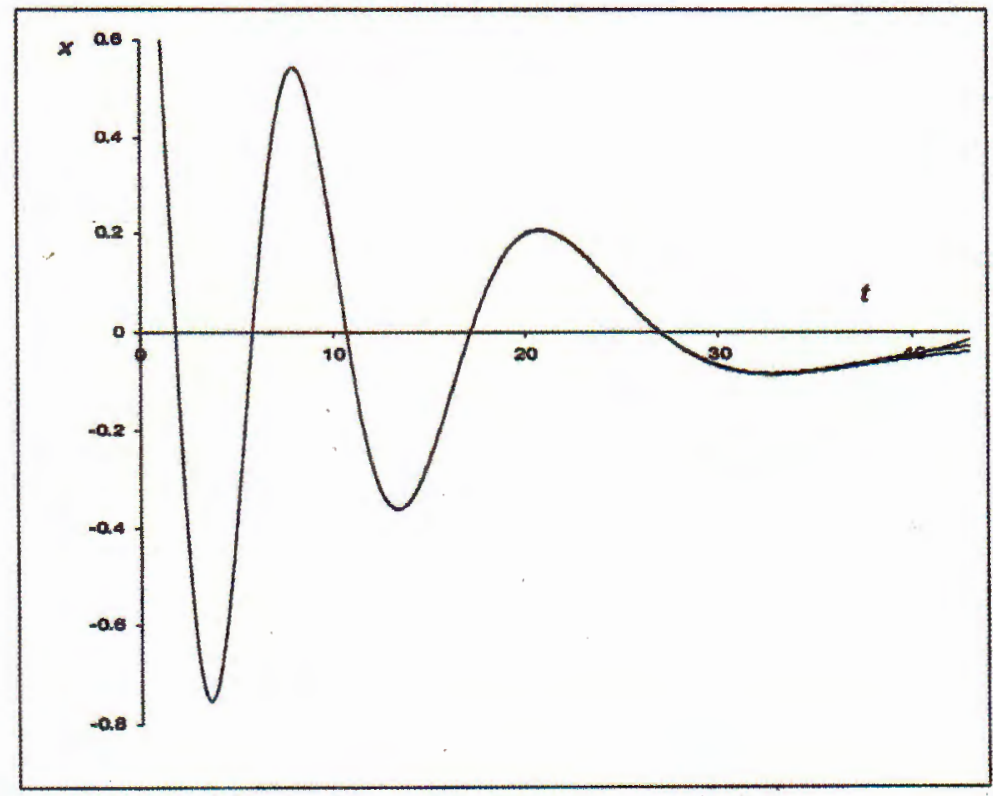

Fig. 1

First and second approximate (mixed type: second approximation is for the linear part and first approximation for the nonlinear part) solution Eq. (3.11) of Eq. (3.3) in which $\psi$ is computed by respectively Eq. (3.15) and Eq. (3.16), for $\varepsilon=0.1$ [with initial conditions, $x(0)=1, \dot{x}(0)=0$ or $a(0)=1.0079, \psi(0)=-0.0738]$ and corresponding numerical solution (generated by Runge-Kutta fourth order procedure) are plotted. Clearly first approximate solution agrees with numerical solutions nicely until $t \leq 38$ and second approximation for $t<41$. When $t>41$, second approximation rapidly changes to upward while first approximation slowly diverges from the numerical solution when $t>38$. It is noted that the middle line (after $t=41$ ) represents the numerical solution. 


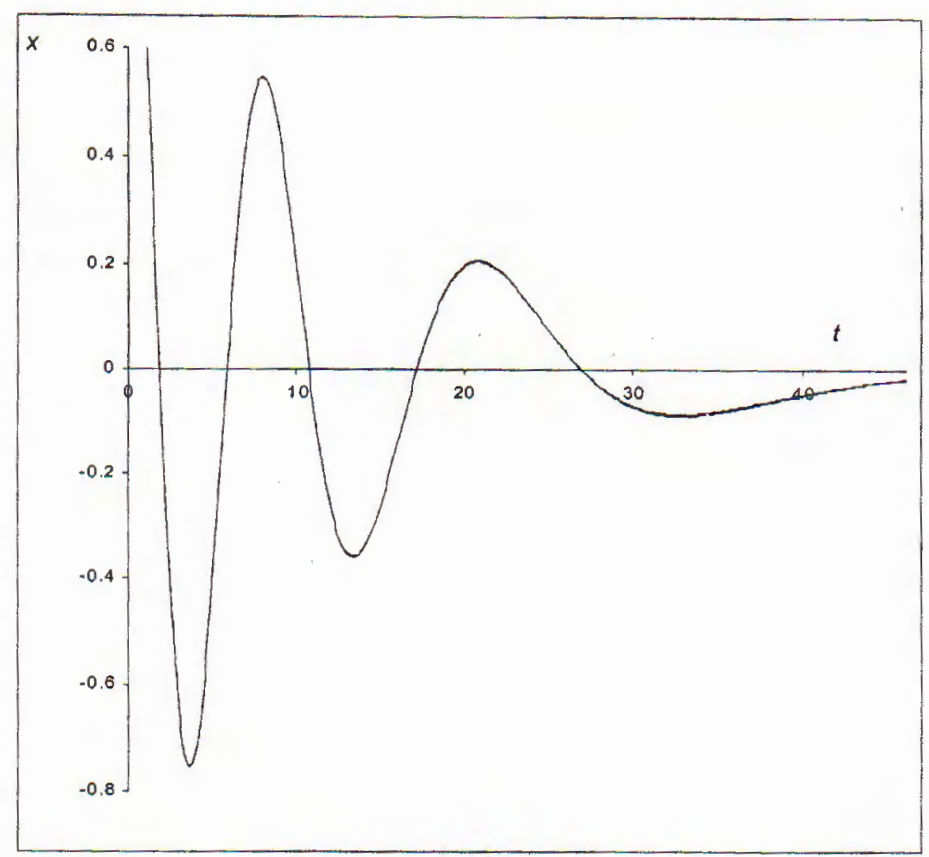

Fig. 2

Second approximate (mixed type: second approximation is for the linear part and first approximation for the nonlinear part) solution Eq. (3.11) of Eq. (3.3) in which $\psi$ is computed by Eq. (3.16) for $\varepsilon=0.1$ [with initial conditions, $x(0)=1, \dot{x}(0)=0$ or $a(0)=$ $1.0079, \psi(0)=-0.0738]$ is plotted when $t \leq 38$, where $x(38)=-0.0605, \dot{x}(38)=0.0071$ and then initialized these values of $x, \dot{x}$, approximate solution Eq. (3.22) is computed and potted from $t=38$ to $t=46$. The corresponding numerical solution is also plotted to compare the results. The figure indicates that the matched solution nicely agrees with the numerical solution.

\section{References}

1. Poincare H. Les Methods Nouvelles de la Mecanique Celeste, Paris, 1892.

2. Wetzel, G. Z. Physik 38 (1926) 518.

3. Kramers H. A., G. Z. Physik 39 (1926) 828.

4. Brillouin L., Compt. Rend. 183 (1926) 24.

5. Feshenko S. F., Shkil N. I. and Nikolenko L. D., Asymptotic Method in the Theory of linear differential Equation, (in Russian), Naukova Dumka, Kiev 1966 [English translation, Amer, Elsevier Publishing Co., INC. New York 1967).

6. Nayfeh A. H., Perturbation Methods, J. Wiley, New York, 1973.

7. Krylov N. N. and N., N., Bogoliubov, Introduction to nonlinear Mechanics. Princeton University presses, New Jersey, 1947.

8. Bogoliubov, N. N. and Yu. Mitropolskii, Asymptotic Methods in the Theory of nonlinear Oscillations, Gordan and Breach, New York, 1961.

9. Mitropolskii Yu., Problems on Asymptotic Methods of non-stationary Oscillations (in Russian), Izdat, Nauka, Moscow, 1964. 
10. Bojadziev G. and Edwards J., On some asymptotic methods for non-oscillatory and oscillatory processes, Nonlinear Vibration Problems 20 (1981) 69-79.

11. Shamsul Alam M., Unified Krylov-Bogoliubov-Mitropolskii method for solving $n$ th order nonlinear systems with slowly varying coefficients, Journal of Sound and Vibration 265 (2003) 987-1002.

12. Shamsul Alam M., Bellal Hossain M. and Shanta S. S., Perturbation theory for damped nonlinear systems with varying coefficients, Indian J. pure and appl. Math. 34 (2003) 1359-1368.

13. Shamsul Alam M., Perturbation theory for damped nonlinear systems with large damping, Indian J. pure and appl. Math. 32 (2001) 1453-1461.

14. Shamsul Alam M., Alam M. F. and Shanta S. S., Approximate solutions of nonoscillatory systems with slowly varying coefficients, Ganit (Bangladesh J. of Math. Soc.) 21 (2001) 55-59.

15. Popov I. P., A generalization of the Bogoliubov asymptotic method in the theory of nonlinear oscillations (in Russian), Dokl. Akad. Nauk. SSSR 111 (1956) 308-310.

16. Cap F. F., Averaging method for the solution of nonlinear differential equations with periodic non-harmonic solutions, Int. J. nonlinear Mech. 9 (1974) 441-450.

17. Bojadziev G. N., Damped oscillating processes in biological and biochemical systems, Bull. Math. Biol. 42 (1980) 701-717.

19. Minorski N., Nonlinear Oscillations, Princeton: Von Nostrand Co., 1962.

20. Hung Cheng and Tai Tsun Wu, An aging spring, Studies in applied Mathematics 49(1970) 183-185.

21. Cole J. D., Perturbation Method in applied Mathematics, Ginn Blaisdell, 1968.

22. Bojadziev G. N., Damped forced nonlinear vibrations of systems with delay, J. Sound and Vibraion 46 (1976) 113-120.

23. Bojadziev G. N., Damped nonlinear oscillations modeled by a 3-dimensional differential system, Acta Mechanica 48 (1983) 193-201.

24. Murty I. S. N., A unified Krylov-Bogoliubov method for solving second order nonlinear systems, Int. J. nonlinear Mech. 6 (1971) 45-53.

Received May 14, 2004

\section{ẢNH HƯỚNG CƯA XẤP Xİ BẬC CAO TRONG NGHIẸM TIỆM CẬN CƯA PHƯONG TRİNH VI PHÂN VÓI HỆ SỐ BIẾN ĐỒI CHÂM TRONG LÂN CÂAN ĐIỂM RẼ் NHÁNH}

Nghiềm xấp xỉ bậc hai của phương trình vi phân cấp hai với tham số biến đởi chậm đã nhận được bằng phương pháp Krưlôv-Bogôliubôv-Mitropôlskii, cùng với minh hoạ trong một ví dụ. Các xấp xỉ bậc hai và bậc cao hơn nữa cho phép ta nhận được nghiệm chính xác hơn xấp xỉ bậc nhất khi mà tần số quy đổi lớn hơn nhiều lần tham số bé. Ngược lại khi tần số quy đổi nhỏ (gần với điểm rẽ nhánh) thì nghiệm xấp xỉ bậc cao lại phân kỳ nhanh hơn các xấp xỉ bậc thấp. Lúc này nghiệm tiệm cận tương thích đóng vai trò quan trọng. Điều này được minh hoạ trong ví dụ. 